\title{
Ventilator Weaning: How Far from the Final Quantum Leap?
}

\author{
Luca Salvatore De Santo ${ }^{1}$. $\cdot$ Antonio Esquinas $^{2}$
}

Received: 4 May 2018 / Accepted: 6 August 2018 / Published online: 9 August 2018

(c) Springer Science+Business Media, LLC, part of Springer Nature 2018

Dear Editor, we read with great interest the paper by Palkar and co-workers [1]. These authors should be commended for their efforts in weaning research. Indeed, this article is part of a larger prospective observational study on the predictive value of serial diaphragm ultrasonography that has been contemporarily published elsewhere [2]. We dare to submit here an overall comment since these two papers received a single-institutional review board approval, analysed the same patients group within the same time frame, underwent a uniform ultrasonographic method and global clinical management, and, finally, speculated on the same outcome measure (extubation failure). In a small series of patients in a medical intensive care unit, this research group serially tracked diaphragm excursion and contraction velocity during weaning process and early after extubation. Further, they developed and evaluated the performance of a new parameter, the excursion-time index, which is a product of diaphragm excursion and inspiratory time. Study methodology is overall sound, but some issues might deserve further comments by the authors. Patients' sample is poorly characterised, and hardly homogeneous. Indeed, reasons for initial mechanical ventilation are not detailed in the two subgroups and patients displayed different potentials for extubation failure (seven patients out of twenty received pre-emptive non-invasive ventilation early after weaning). Provokingly, a reader might argue that this limited subgroup might prove more clinically intriguing and best suited for extensive research. More, there are no hints on the reasons for reintubation. As expected, the results of these observations are largely confirmative of previous evidence: diaphragmatic ultrasound parameters,

Luca Salvatore De Santo

luca.desanto@unifg.it

Antonio Esquinas

antmesquinas@gmail.com

1 University of Foggia, and Clinica Montevergine, GMV Health Care and Research, Via Mario Malzoni 5, 83013 Mercogliano, AV, Italy

2 Hospital Morales Meseguer, Avenida Marques de Los Velez s/n, 30008 Murcia, Spain either conventional or innovative, are imperfect predictors of extubation outcomes. The clinical bottom line, simply and sobering, remains that, since weaning failure is multifactorial in nature, it is naïve to think that a single parameter might predict the fate of a complex interplay. As described in methods section, diaphragmatic ultrasound monitoring was not factored in the decision making for weaning and subsequent indication for non-invasive support or reintubation. Methodology or limitation? A dichotomy is clear; currently, management algorithms fail to safely wean from MV as many as $20-30 \%$ of patients, but research programs still focus on a single, increasingly sophisticated, "salami slice" with no real potential for clinical breakthrough. Long ago, a seminal editorial asked "Is weaning an art or a science?" [3]. Maybe, the answer is simply both, if we totally commit to a patient-tailored approach and stick with a thorough, holistic, innovative look.

\section{Compliance with Ethical Standards}

Conflict of interest There is no conflict of interest to disclose.

Informed Consent Informed consent and animal welfare are not applicable.

\section{References}

1. Palkar A, Mayo P, Singh K, Koenig S, Narasimhan M, Singh A, Darabaner R, Greenberg H, Gottesman E (2018) Serial diaphragm ultrasonography to predict successful discontinuation of mechanical ventilation. Lung Mar. https://doi.org/10.1007/s0040 8-018-0106-xL

2. Palkar A, Narasimhan M, Greenberg H, Singh K, Koenig S, Mayo P, Gottesman E (2018) Diaphragm excursion-time index: a new parameter using ultrasonography to predict extubation outcome. Chest. https://doi.org/10.1016/j.chest.2018.01.007

3. Milic-Emili J (1986) Is weaning an art or a science? Am Rev Respir Dis 134(6):1107-1108 